\title{
Avaliação da influência do tipo facial nos tamanhos dos espaços aéreos nasofaríngeo e bucofaríngeo*
}

Aline Maria Alencar de Castro ${ }^{\star \star}$, Maria Helena Ferreira Vasconcelos ${ }^{\star \star \star}$

\begin{abstract}
Resumo
Introdução: a diminuição no tamanho do espaço aéreo nasofaríngeo, causada pela hipertrofia da tonsila faríngea, tem sido associada a alterações no padrão normal de crescimento craniofacial e a efeitos deletérios na oclusão. Objetivos: avaliar variações nos tamanhos dos espaços aéreos nasofaríngeo e bucofaríngeo de acordo com o padrão de crescimento craniofacial, a correlação entre os tamanhos dos espaços e o índice VERT, além de verificar um possível dimorfismo sexual. Métodos: foi utilizado um total de 90 telerradiografias laterais de pacientes, divididos em três grupos: meso, braqui e dolicofacial, determinados por meio do índice VERT de Ricketts. Os pacientes da amostra, com idades entre 9 e 16 anos, apresentavam padrão respiratório nasal, sem qualquer tipo de obstrução. Resultados: não foi verificada variação estatisticamente significante nos tamanhos dos espaços aéreos nasofaríngeo e bucofaríngeo, quando comparados os três tipos faciais. Também não foi encontrada correlação entre os tamanhos dos espaços aéreos e os valores do índice VERT de Ricketts dos pacientes e não houve dimorfismo sexual. Conclusões: pode-se descartar a influência do tipo facial nos tamanhos dos espaços aéreos nasofaríngeo e bucofaríngeo.
\end{abstract}

Palavras-chave: Espaço nasofaríngeo. Espaço bucofaríngeo. Padrão de crescimento craniofacial. Índice VERT de Ricketts.

\section{INTRODUÇÃO}

O tamanho do espaço nasofaríngeo pode apresentar-se alterado devido à presença da adenóide (tonsila faríngea hipertrófica); e o bucofaríngeo em função da postura inadequada da língua, causada pelo desequilíbrio neuromuscular promovido pela respiração bucal ${ }^{6,10}$. Pacientes portadores destes distúrbios funcionais quase sempre são respiradores bucais.

O padrão de crescimento dolicofacial tem sido, freqüentemente, associado ao paciente respirador bucal, supostamente por este apresentar o espaço aéreo mais estreito; porém, tal fato não está suportado por evidências científicas. Parte dos estudiosos do assunto ressalta que, quando o tamanho do espaço nasofaríngeo apresenta-se diminuído - seja pela presença da adenóide ou pela estrutura anatômica estreita da nasofaringe - o paciente apresenta uma alteração no padrão normal de crescimento e desenvolvimento craniofacial, representada por uma tendência de crescimento mais vertical da face ${ }^{8}$. Algumas pesquisas relatam que os pacientes dolicofa-

* Resumo da dissertação de mestrado apresentada à Faculdade de Odontologia da UMESP para a obtenção do título de mestre em Ortodontia.

** Mestre pelo programa de pós-graduação em Odontologia (área de concentração Ortodontia) da UMESP. *** Professora titular do programa de pós-graduação em Odontologia (área de concentração Ortodontia) da UMESP. Doutora em Ortodontia pela
FOB-USP. 
ciais são os mais propensos a desenvolver respiração bucal, por apresentarem estruturas nasais e nasofaríngeas mais estreitas que os demais tipos faciais, ${ }^{6,15}$. Nem todos, porém, concordam com esta relação direta de causa-efeito, sugerindo que a adenóide e outros fatores ambientais são predominantes na determinação das características craniofaciais ${ }^{10,14,28}$. O que ocorre, provavelmente, é a interação de fatores genéticos e ambientais na determinação do padrão de crescimento craniofacial ${ }^{19}$.

A largura ântero-posterior da nasofaringe é estabelecida precocemente, nos primeiros anos de vida $9,13,23$. A partir dos dois anos, o aumento na largura ântero-posterior ou sagital da nasofaringe, pelo crescimento na sincondrose esfeno-occipital, seria minimizado pelo crescimento anterior da primeira vértebra cervical. $\mathrm{O}$ tamanho e a configuração da nasofaringe podem estar associados à obstrução aérea pela adenóide ${ }^{13}$.

O crescimento excessivo, ou hipertrofia, da tonsila faríngea também pode causar obstrução na nasofaringe. Esta obstrução somente estará presente se a adenóide apresentar um crescimento desequilibrado em relação ao aumento de tamanho da nasofaringe $e^{4,23}$. A tonsila faríngea cresce rapidamente nos primeiros anos de vida e, aproximadamente, aos 3 anos de idade ela ocupa quase metade da nasofaringe. A partir desta idade, tanto a adenóide como a nasofaringe continuam a crescer, só que em velocidade menor. $\mathrm{O}$ pico de crescimento da adenóide pode ocorrer mais cedo, aos 10-11 anos, ou mais tarde, aos 14-15 anos. A partir de então, a adenóide começa a involuir em tamanho, aumentando, desta forma o tamanho do espaço nasofaríngeo. O tamanho adulto deste espaço pode ser atingido mais precocemente, aos 13 anos $^{3}$, ou mais tardiamente, aos 15-16 anos ${ }^{28,30}$.

Essas considerações despertaram a curiosidade dos autores do presente trabalho, que objetivaram verificar a afirmativa a respeito da variação do tamanho dos espaços aéreos, especialmente em relação aos tipos faciais em pacientes sem distúrbio funcional respiratório.

\section{MATERIAL E MÉTODOS Material}

A amostra deste estudo constituiu-se de telerradiografias cefalométricas laterais de arquivo pertencentes a 90 indivíduos, sendo 55 do gênero masculino e 35 do gênero feminino, com idades entre 9 e 16 anos, selecionados para tratamento ortodôntico na clínica de pós-graduação do departamento de Ortodontia da Universidade Metodista de São Paulo.

Como critério de seleção da amostra, os pacientes deveriam ser, predominantemente, respiradores nasais, não apresentar ausências dentárias e não ter sido submetidos a tratamentos ortodôntico ou cirúrgico prévios.

\section{Métodos}

Avaliação do modo respiratório

Os pacientes, após exame otorrinolaringológico prévio, responderam a um questionário, no qual relataram serem respiradores predominantemente nasais. Os pacientes que relataram ser respiradores predominantemente bucais foram excluídos da amostra.

Em seguida, a confirmação do modo respiratório dos pacientes foi feita utilizando-se o espelho nasal milimetrado de Altmann, abaixo das narinas do paciente, para análise do fluxo aéreo nasal. Este espelho registrou a presença ou ausência de fluxo aéreo nasal ou bucal.

\section{Determinação do tipo facial}

Para a determinação do tipo facial, foi utilizado o índice VERT da análise cefalométrica de Ricketts $^{19}$. Os pontos cefalométricos dessa análise foram obtidos utilizando-se o programa Radiocef 4.0 Radio Memory Informática.

Ricketts et al. ${ }^{19}$ estabeleceram três tipos básicos de crescimento facial: mesofacial (crescimento equilibrado), dolicofacial (crescimento predominantemente vertical) e braquifacial (crescimento predominantemente horizontal). Os autores criaram o índice VERT, que indica a quantidade 
de crescimento vertical da face. Também determinaram padrões de normalidade e os respectivos desvios-padrão, utilizando crianças caucasianas de nove anos de idade. Então, estabeleceram valores médios anuais de mudança nos valores de cada ângulo, resultante do crescimento.

$\mathrm{Na}$ determinação do índice VERT, obtidos os valores dos ângulos do paciente, calcula-se a norma individualizada para cada ângulo, levando em consideração a idade do indivíduo. Após estabelecida a diferença entre o valor encontrado e a norma individualizada, divide-se o valor encontrado pelo desvio clínico (que varia de acordo com o ângulo). Atribui-se um sinal positivo quando o valor indicar uma tendência braquifacial de crescimento, ou negativo quando o valor encontrado indicar uma tendência dolicofacial. O índice VERT é calculado pela média aritmética dos cinco valores de classificação facial.

Se o índice VERT encontrado for muito negativo (valores iguais ou menores que $-0,5$ ), o paciente é classificado como dolicofacial. No caso deste valor ser igual ou maior que $+0,5$, o paciente é classificado como braquifacial. Aqueles pacientes com índice VERT entre $-0,5$ e $+0,5$ são classificados como mesofaciais.

Os pacientes da amostra foram, então, divididos em três grupos, com 30 pacientes cada, de

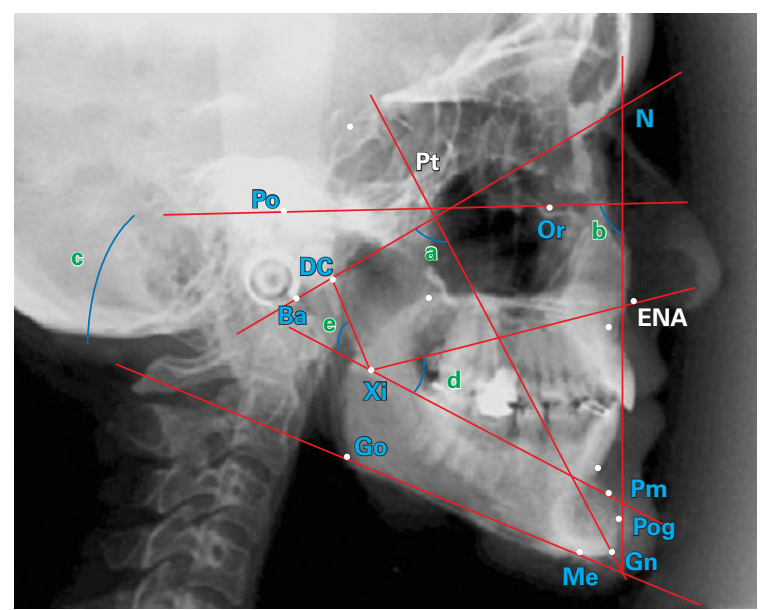

FIGURA 1 - Medidas angulares da análise de Ricketts ${ }^{19}$, utilizadas para a determinação do tipo facial. acordo com o tipo facial: mesofaciais, dolicofaciais e braquifaciais.

Medidas angulares para a determinação do tipo facial (Fig. 1)

a) Ângulo do eixo facial: (N-Ba).(Pt-Gn).

b) Ângulo facial ou profundidade facial: (PoOr).(N-Pog).

Or).

c) Ângulo do plano mandibular: (Go-Me).(Po-

d) Altura da face inferior: (Xi-ENA). (Xi-Pm).

e) Arco mandibular: (DC-Xi).(Xi-Pm).

\section{Demarcação dos espaços naso e bucofaríngeo}

Os traçados dos espaços aéreos nasofaríngeo e bucofaríngeo foram realizados, manualmente, em um ambiente escurecido. Foram avaliadas as distâncias entre a borda posterior da naso e bucofaringe e o palato mole para definir o tamanho do espaço aéreo nos pacientes respiradores nasais com padrão de crescimento do tipo meso, braqui e dolicofaciais.

Pontos cefalométricos para análise dos espaços nasofaríngeo e bucofaríngeo (Fig. 2)

- Sela (S): situado no centro geométrico do contorno da sela túrcica.

- Básio (Ba): ponto mais inferior do osso occi-

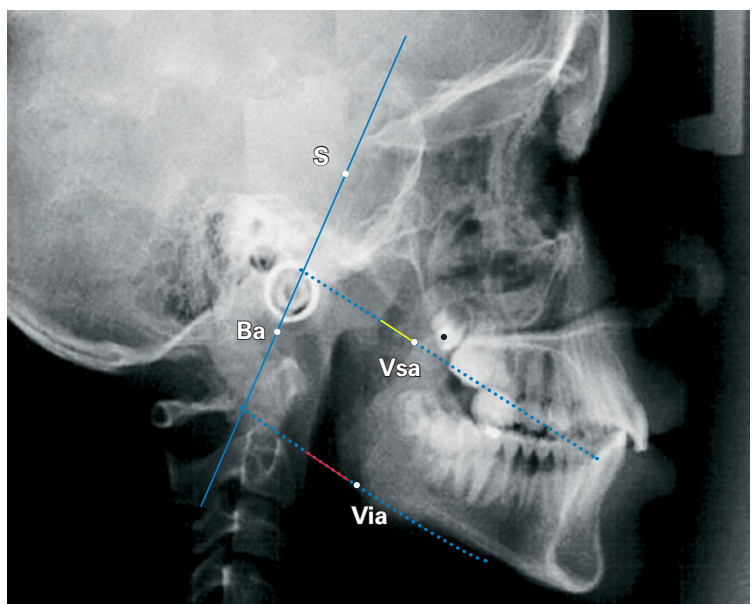

FIGURA 2 - Medidas lineares dos espaços nasofaríngeo e bucofaríngeo, conforme Zanelato ${ }^{32}$. 
pital, na margem anterior do forame magno.

- Via aérea súpero-anterior (Vsa): localizado na metade anterior do palato mole, na porção mais próxima da parede posterior da nasofaringe ${ }^{16}$.

- Via aérea ínfero-anterior (Via): situado na união da borda inferior da mandíbula com a borda posterior da língua ${ }^{16}$.

Após a determinação destes pontos, foi traçada uma linha unindo os pontos Sela e Básio (S-Ba), que foi usada como referência para mensuração das vias aéreas superior e inferior. A partir desta linha, foram traçadas linhas perpendiculares que passavam pelos pontos Vsa (via aérea súperoanterior) e Via (via aérea ínfero-anterior) (Fig. 2). Foram obtidas, portanto, as linhas:

- SBa-Vsa: perpendicular à linha SBa, passando pelo ponto Vsa.

- SBa-Via: perpendicular à linha SBa, passando pelo ponto Via.

Para a determinação dos tamanhos dos espaços aéreos, foram mensurados os segmentos de reta na região da naso e bucofaringe, sem incluir o palato mole. Os valores normais para o espaço nasofaríngeo são de $12 \mathrm{~mm}$ para pacientes na fase de dentadura mista e $17,4 \mathrm{~mm}$ para pacientes na dentadura permanente ${ }^{18}$. Quando este espaço apresentar valores menores ou iguais a $5 \mathrm{~mm}$, há um indicativo de obstrução nasal. Os valores normais para o espaço bucofaríngeo variam de 10 a $12 \mathrm{~mm}$ para qualquer idade ${ }^{18}$. Para valores maiores que $15 \mathrm{~mm}$, há indícios do posicionamento anteriorizado da língua. $\mathrm{Na}$ metodologia adotada no presente trabalho, utilizou-se como base os valores normativos preconizados por McNamara Jr. ${ }^{16}$, porém o método utilizado foi o preconizado por Zanelato $^{32}$, o qual ofereceu maior confiabilidade de medição, em virtude de ter como referência um plano localizado na base do crânio.

\section{Erro do método}

$\mathrm{Na}$ análise do erro sistemático, foram remedidos $20 \%$ da amostra inicial, o que equivale a 18 radiografias. Utilizou-se o teste $\mathrm{t}$ pareado e foi adotado o nível de significância de 5\%. Para o erro casual, calculou-se o erro proposto por Dahlberg.

\section{Método estatístico}

$\mathrm{Na}$ análise dos dados, utilizou-se a estatística descritiva com parâmetros de média e desvio-padrão. Para comparação dos valores dos espaços nasofaríngeo e bucofaríngeo entre os padrões faciais dólico, meso e braqui, e entre os gêneros, foi utilizada a análise de variância a dois critérios, modelo fixo.

Para verificar se os dados obedeciam aos critérios de normalidade e homocedasticidade (homogeneidade de variância), o que permitiria o uso da análise de variância, utilizou-se os testes de Kolmogorov-Smirnov e de Bartlett.

Para verificar se houve correlação entre os valores do índice VERT e os valores dos espaços nasofaríngeo e bucofaríngeo, foi utilizado o coeficiente de correlação de Pearson.

Em todos os testes estatísticos foi adotado nível de significância de 5\% ( $<<0,05)$.

\section{RESULTADOS}

Os resultados do teste de Kolmogorov-Smirnov não mostraram desvio estatisticamente significante da normalidade para os dados dos espaços nasofaríngeo e bucofaríngeo em nenhum dos grupos ( $p>$ $0,05)$. O teste de Bartlett também mostrou que a diferença na variabilidade entre os grupos não foi estatisticamente significante $(p=0,213)$. Assim foi possível a utilização da análise de variância a dois critérios. As tabelas 1 e 2 demonstram, respectivamente, a média e o desvio-padrão para os tamanhos dos espaços nasofaríngeo e bucofaríngeo.

A análise de variância mostrou não haver diferença estatisticamente significante entre padrão facial, gênero ou interação entre estes fatores, tanto para o espaço naso como para o bucofaríngeo (Tab. 3,4 ).

O coeficiente de correlação de Pearson mostrou não haver correlação estatisticamente significante entre o índice VERT e os espaços nasofaríngeo e bucofaríngeo ( $\mathrm{r}=-0,06 ; \mathrm{p}=0,593)$, conforme ilustrado nos gráficos 1 e 2 . 


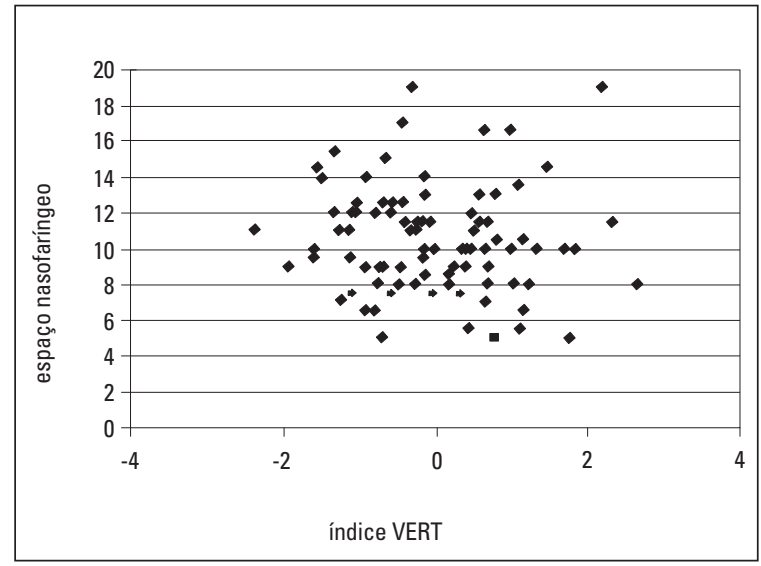

GRÁFICO 1 - Distribuição dos valores do índice VERT e do espaço nasofaríngeo dos 90 indivíduos da amostra.

Tabela 1 - Média e desvio-padrão do espaço nasofaríngeo, por padrão facial e gênero.

\begin{tabular}{ccccccc}
\multirow{2}{*}{ padrão } & \multicolumn{2}{c}{ masc. (M) } & \multicolumn{2}{c}{ fem. (F) } & \multicolumn{2}{c}{$M+F$} \\
\cline { 2 - 7 } & média & d.p. & média & d.p. & média & d.p. \\
dólico (D) & 10,20 & 2,95 & 10,90 & 2,59 & 10,55 & 2,75 \\
meso (M) & 10,28 & 2,56 & 10,71 & 3,16 & 10,45 & 2,77 \\
braqui (B) & 11,08 & 2,15 & 10,09 & 4,08 & 10,52 & 3,37 \\
D+M+B & 10,48 & 2,56 & 10,53 & 3,33 & 10,51 & 2,94 \\
\hline
\end{tabular}

Tabela 3 - Análise de variância a dois critérios (padrão facial e gênero) para comparação das diferenças no espaço nasofaríngeo.

\begin{tabular}{rccccccc} 
& $\mathrm{GL}$ & $\mathrm{QM}$ & $\mathrm{GL}$ & $\mathrm{QM}$ & & $\mathrm{p}$ \\
\cline { 2 - 5 } efeito & efeito & efeito & erro & erro & & \\
padrão & 2 & 0,0597 & 84 & 9,0402 & 0,007 & 0,993 n.s. \\
gênero & 1 & 0,0493 & 84 & 9,0402 & 0,005 & 0,941 n.s. \\
interação & 2 & 6,0796 & 84 & 9,0402 & 0,672 & 0,513 n.s.
\end{tabular}

n.s. - diferença estatisticamente não significante.

\section{DISCUSSÃO}

A diminuição do tamanho do espaço aéreo nasofaríngeo tem sido freqüentemente associada à hipertrofia da tonsila faríngea, o que pode resultar em uma obstrução nasal $1^{9,14,23,24,28}$. O tamanho da adenóide deverá estar extremamente aumentado para afetar o tamanho do espaço aéreo nasal ${ }^{28}$. A associação entre a presença de adenóide e a obstrução nasal pode não ser observada, visto que a maior parte dos pacientes da amostra de um estudo que

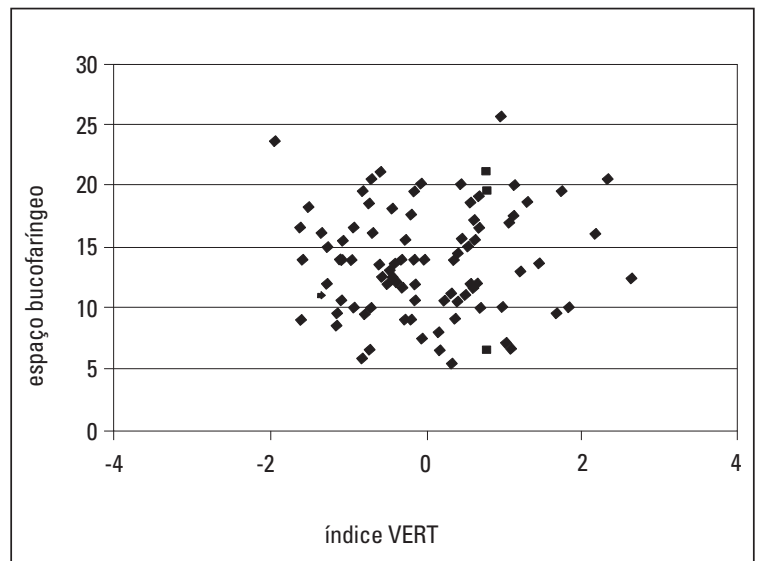

GRÁFICO 2 - Distribuição dos valores do índice VERT e do espaço bucofaríngeo dos 90 indivíduos da amostra.

Tabela 2 - Média e desvio-padrão do espaço bucofaríngeo, por padrão facial e gênero.

\begin{tabular}{ccccccc}
\multirow{2}{*}{ padrão } & \multicolumn{2}{c}{ masc. (M) } & \multicolumn{2}{c}{ fem. (F) } & \multicolumn{2}{c}{$M+F$} \\
\cline { 2 - 7 } & média & d.p. & média & d.p. & média & d.p. \\
dólico (D) & 13,63 & 4,52 & 13,90 & 4,34 & 13,77 & 4,35 \\
meso (M) & 13,19 & 4,42 & 11,88 & 2,80 & 12,67 & 3,86 \\
braqui (B) & 15,08 & 4,37 & 14,44 & 5,23 & 14,72 & 4,81 \\
D+M+B & 13,87 & 4,41 & 13,56 & 4,41 & 13,72 & 4,39
\end{tabular}

Tabela 4 - Análise de variância a dois critérios (padrão facial e gênero) para comparação das diferenças no espaço bucofaríngeo.

\begin{tabular}{rcccccc}
\multirow{2}{*}{ efeito } & $\mathrm{GL}$ & $\mathrm{QM}$ & $\mathrm{GL}$ & $\mathrm{QM}$ & \multirow{2}{*}{$\mathrm{f}$} & $\mathrm{p}$ \\
\cline { 2 - 5 } & efeito & efeito & erro & erro & & \\
padrão & 2 & 36,144 & 84 & 19,468 & 1,857 & 0,163 n.s. \\
gênero & 1 & 6,9886 & 84 & 19,468 & 0,359 & 0,551 n.s. \\
interação & 2 & 4,6594 & 84 & 19,468 & 0,239 & 0,788 n.s. \\
\hline
\end{tabular}

n.s. - diferença estatisticamente não significante.

apresentava hipertrofia das tonsilas faríngeas não era de respiradores bucais ${ }^{25}$. Quanto maior o tamanho do espaço aéreo maior a porcentagem de respiração nasal, havendo uma relação direta ${ }^{30}$.

A respiração bucal causada pela diminuição do tamanho do espaço nasofaríngeo pode, também, ser o resultado de uma predisposição anatômica (espaço aéreo estreito) ${ }^{6}$. Na presença de uma nasofaringe estreita, uma massa pequena de tecido adenóideo já seria suficiente para causar uma obs- 
trução da passagem aérea. Em contrapartida, a respiração bucal associa-se não somente à diminuição do tamanho do espaço nasofaríngeo, mas também a outros fatores como rinites, amigdalites, bronquites e outros ${ }^{11}$. Os pacientes dolicofaciais são os mais propensos a desenvolver respiração bucal, por apresentarem estruturas nasais e nasofaríngeas mais estreitas que os demais tipos faciais ${ }^{6,15}$. Isto não foi confirmado no presente trabalho, pois não foram encontradas diferenças estatisticamente significantes nos tamanhos dos espaços nasofaríngeo e bucofaríngeo, quando da comparação dos três tipos faciais. De acordo com a tabela 1, o grupo de pacientes dolicofaciais apresentou média de $10,55 \mathrm{~mm}$ para o tamanho do espaço nasofaríngeo, o de mesofaciais teve média de $10,45 \mathrm{~mm}$ e o de braquifaciais média de $10,52 \mathrm{~mm}$.

Em estudos que avaliaram pacientes respiradores nasais, verificou-se que o tamanho do espaço aéreo nasal pode variar (em crianças de 9 a 11 anos) de $0,32 \mathrm{~mm}^{2}$, segundo Ellingsen et al. ${ }^{5}$, a $0,4 \mathrm{~mm}^{2}$, segundo Crouse et al. ${ }^{3}$; ou $0,46 \mathrm{~mm}^{2}$, segundo Fields et al. ${ }^{8}$, em jovens de 11 a 17 anos, o que pode ser explicado pela idade da amostra de estudo.

O crescimento máximo da adenóide ocorre entre 3 e $7 \operatorname{anos}^{9,14}$. Ele pode, ainda, estar presente aos 10-11 anos ou aos 14-15 anos ${ }^{23,24}$. O tamanho adulto do espaço aéreo nasal pode ser atingido mais precocemente, aos 13 anos $^{3}$, ou mais tardiamente, aos 15-16 anos ${ }^{28,29}$. O valor médio do espaço aéreo nasofaríngeo encontrado na presente pesquisa foi de $10,51 \mathrm{~mm}$, superior aos valores encontrados em outros estudos, que variaram de $6,8 \mathrm{~mm}^{22}$ a $9,5 \mathrm{~mm}^{32}$. A maior diferença pode ser explicada pelas diferentes faixas etárias utilizadas nas pesquisas.

Nos períodos de pré e pós-puberdade, o aumento no tamanho do espaço nasofaríngeo ocorre devido ao assincronismo entre o crescimento da nasofaringe (que cresce muito pouco após a infância) e, principalmente, o decréscimo no crescimento da adenóide que ocorre neste período ${ }^{9,18}$. Este fator seria, em parte, responsável pela peque- na porcentagem de pacientes com obstrução aérea nesta fase $\mathrm{f}^{6,23}$.

As dimensões ideais para o espaço aéreo nasofaríngeo em pacientes com padrão facial harmonioso seriam de $17,4 \mathrm{~mm}$ para pacientes adultos e $12 \mathrm{~mm}$ para pacientes na fase de dentadura mista. Para o espaço bucofaríngeo, a norma seria $10-12 \mathrm{~mm}$ e não sofreria muita alteração com o passar da idade ${ }^{16}$. Um espaço nasofaríngeo de $4 \mathrm{~mm}$ ou mais forneceria espaço suficiente para a passagem aérea adequada ${ }^{20}$. Em respiradores nasais, aos 7 anos, $47 \%$ da profundidade total da nasofaringe está livre para exercer a função respiratória normal ${ }^{22}$. Uma porcentagem de 49,28\% foi encontrada em 8 crianças com idades entre 6,1 e 9,11 anos $^{18}$. Por outro lado, nem sempre é possível determinar qual tamanho da adenóide é suficiente para causar obstrução do espaço nasofaríngeo. É preciso analisar a relação que o tecido adenoideano guarda com a nasofaringe $e^{4,7}$.

Ao contrário de outros estudos 22,32 , na presente pesquisa, não foi encontrada diferença estatisticamente significante nos tamanhos dos espaços aéreos nasofaríngeo e bucofaríngeo entre os gêneros masculino e feminino.

Em decorrência da face alongada, característica do paciente respirador bucal, esta tem sido, algumas vezes, erroneamente descrita como a "síndrome da face longa". Apesar desta associação estar incorreta, alguns autores observaram um crescimento craniofacial mais vertical nos pacientes portadores deste distúrbio respiratório ${ }^{8,11}$. A grande maioria descarta a total influência de um fator ambiental na forma de crescimento da face ${ }^{11,21,26,27}$. A genética seria o fator primordial na determinação do padrão de crescimento craniofacial, podendo esta ser influenciada ou não por fatores ambientais. Apesar de ser observado aumento na AFAI, aumento na inclinação do plano mandibular e hipotonia da musculatura orofacial em crianças com tonsilas hipertróficas, a influência desta obstrução nasofaríngea na direção de crescimento craniofacial pode não ser observada ${ }^{26}$. 
Em estudos longitudinais, é comum a observação da mudança no modo respiratório de nasal para bucal e vice-versa, ao longo do tempo ${ }^{21}$. O crescimento e desenvolvimento craniofacial se estabelece precocemente e não é influenciado de forma significante pelo modo respiratório ${ }^{21,27}$.

Avaliando apenas pacientes respiradores nasais, o tamanho do espaço aéreo nasofaríngeo pode não exercer influência na morfologia craniofacial ${ }^{17,22}$. O espaço nasofaríngeo precisa apenas ser suficiente para permitir a passagem aérea nasal ${ }^{22}$. Por meio da mensuração da resistência aérea nasal, uma correlação significante entre esta e a morfologia craniofacial pode não ser encontrada ${ }^{31}$. A resistência aérea nasal e o padrão respiratório nasal podem não apresentar correlação significante com a configuração morfológica das estruturas craniofaciais ${ }^{31}$.

O espaço bucofaríngeo, ao contrário do nasofaríngeo, não é muito avaliado nos trabalhos científicos. A presente pesquisa encontrou valores similares para o espaço bucofaríngeo, quando comparados os tipos faciais meso, braqui e dolicofacial. Quando comparados pacientes normo e hiperdivergentes, também não se encontraram diferenças significantes na medida do espaço bucofaríngeo ${ }^{12}$. Um detalhe importante do trabalho de Joseph et al. ${ }^{12}$ é que não foi avaliado o padrão respiratório dos pacientes, indicando a provável presença de respiradores bucais na amostra.

Esta pesquisa justifica-se pela escassez de trabathos direcionados à mensuração dos espaços aéreos nasofaríngeo e bucofaríngeo utilizando apenas pacientes predominantemente respiradores nasais, sem qualquer distúrbio funcional respiratório. Os pacientes respiradores bucais são os mais prevalentes neste tipo de estudo. Estes pacientes não foram incluídos na amostra, em virtude da influência que o tamanho da adenóide poderia exercer no tamanho do espaço nasofaríngeo, podendo mascarar os resultados quando fossem comparados os três tipos faciais: meso, braqui e dolicofacial.

\section{CONCLUSÕES}

De acordo com a metodologia empregada neste trabalho e com a análise dos resultados, concluiu-se que:

- Não foi observada influência do padrão de crescimento craniofacial nos tamanhos dos espaços aéreos nasofaríngeo e bucofaríngeo.

- Não houve correlação entre os valores do índice VERT de Ricketts e os tamanhos dos espaços aéreos nasofaríngeo e bucofaríngeo.

- Não houve dimorfismo sexual nos tamanhos dos espaços aéreos nasofaríngeo e bucofaríngeo.

\title{
Evaluation of the influence of facial growth on the sizes of nasopharyngeal and oropharyngeal spaces
}

\begin{abstract}
Introduction: The variation in sizes of the nasopharyngeal and oropharyngeal space occurs due to genetic and/or environmental factors. The reduction in size of the nasopharyngeal space, caused by the hypertrophy of the pharyngeal tonsil, have been associated to alterations in facial growth pattern and to harmful effects in occlusion. Aim: To evaluate variations in size of the nasopharyngeal and oropharyngeal space according to facial growth pattern, to correlate the size of these spaces to the VERT index and to verify a possible sexual dimorphism. Methods: A total of 90 lateral cephalometric radiographs of patients, divided into three groups (mesofacial, dolichofacial and brachyfacial, determined by the VERT index) were used. The patients of the sample, with ages between 9 and 16 years old, had a nasal respiratory mode, without any kind of nasal obstruction. Results: The results did not show statistically significant variation in the size of nasopharyngeal and oropharyngeal, when compared the three facial growth pattern. Besides, there was no correlation between the size of the spaces and the VERT index and a sexual dimorphism was not observed. Conclusions: The conclusions discarded the influence of facial type in the sizes of nasopharyngeal and oropharyngeal spaces.
\end{abstract}

Key words: Nasopharyngeal space. Oropharyngeal space. Craniofacial growth. Ricketts VERT index. 


\section{REFERÊNCIAS}

1. ALCAZAR, N. M. P. V. et al. Estudo cefalométrico naso e bucofaríngeo nas más oclusões Classe I e Classe II divisão I, sem tratamento ortodôntico, com diferentes padrões de crescimento. Rev. Dental Press Ortodon. Ortop. Facial, Maringá, v. 9, n. 4, p. 68-76, jul./ago. 2004.

2. BIZETTO, M. S. P. et al. Estudo cefalométrico comparativo entre crianças respiradoras bucais e nasais nos diferentes tipos faciais. Rev. Dental Press Ortodon. Ortop. Facial, Maringá, v. 9, n. 1, p. 79-87, jan./fev. 2004.

3. CROUSE, U. et al. A longitudinal study of nasal airway size from age 9 to age 13. Angle Orthod., Appleton, v. 69, no. 5, p. 413-418, May 1999.

4. DIAMOND, O. Tonsils and adenoids: why the dilemma? Am. J. Orthod., St. Louis, v. 78, no. 5, p. 495-503, May 1980.

5. ELLINGSEN, R. et al. Temporal variation in nasal and oral breathing in children. Am. J. Orthod. Dentofacial Orthop., St. Louis, v. 107, no. 4, p. 411-417, Apr. 1995.

6. EMSLIE, R. D.; MASSLER, M.; ZWEMER, J. D. Mouth breathing: I. Etiology and effects. J. Am. Dent. Assoc., Chicago, v. 44, p. 506-521, May 1952

7. FAIRCHILD, R. C. A pediatrician views the tonsils and adenoid problem. Am. J. Orthod., St. Louis, v. 54, no. 7, p. 491-494, July 1968.

8. FIELDS, H. W. et al. Relationship between vertical dentofacial morphology and respiration in adolescents. Am. J. Orthod. Dentofacial Orthop., St. Louis, v. 99, no. 2, p. 147-154, Feb. 1991.

9. HANDELMAN, C. S.; OSBORNE, G. Growth of the nasopharynx and adenoid development from one to eighteen years. Angle Orthod., Appleton, v. 46, no. 3, p. 243-259, July 1976.

10. HARVOLD, E. P.; CHIERICI, G.; VARGEVIK, K. Primate experiments on oral sensation and dental malocclusions. Am. J. Orthod., St. Louis, v. 63, no. 5, p. 494-508, May 1972.

11. JABUR, L. B. et al. Estudo clínico da correlação entre padrão respiratório e alterações ortodônticas e miofuncionais. Rev. Odontol. UNICID., São Paulo, v. 9, n. 2, p. 105-117, jul./dez. 1997.

12. JOSEPH, A. A. et al. A cephalometric comparative study of the soft tissue dimensions in persons with hiperdivergent and normodivergent facial patterns. J. Oral Maxillofac. Surg., Philadelphia, v. 56, no. 2, p. 135-139, Feb. 1998.

13. KING, E. W. A roentgenographic study of pharyngeal growth. Angle Orthod., Appleton, v. 22, no. 1, p. 23-36, Jan. 1952.

14. LINDER-ARONSON, S.; LEIGHTON, B. C. A longitudinal study of the development of the posterior nasopharyngeal wall between 3 and 16 years of age. Eur. J. Orthod., Oxford, v. 5, no. 1, p. 47-58, Feb. 1983.

15. MASSLER, M.; ZWEMER, J. D. Mouth breathing II. Diagnosis and treatment. J. Am. Dent. Assoc., Chicago, v. 46, no. 6, p. 658-671, June 1953.

16. McNAMARA JR., J. A. A method of cephalometric evaluation. Am. J. Orthod., St. Louis, v. 86, no. 6, p. 449-469, June 1984.

17. MERGEN, D. C.; JACOBS, R. M. The size of the nasopharynx associated with normal occlusion and Class II malocclusion. Angle Orthod., Appleton, v. 40, no. 4, p. 342-346, Oct. 1970.
18. POOLE, M. N.; ENGEL, G. A.; CHACONAS, S. J. Nasopharyngeal cephalometrics. J. Oral Surg., Chicago, v. 49, no. 3, p. 266-271, Mar. 1980.

19. RICKETTS, R. M. et al. Técnica bioprogressiva de Ricketts. Buenos Aires: Panamericana, 1983.

20. SANTOS PINTO, A.; MONNERAT, M. E. Alterações nasofaringeanas e craniofaciais em pacientes com adenóides hipertróficas: estudo cefalométrico. Rev. Gaúcha Odontol., Porto Alegre, v. 34, n. 4, p. 349-354, abr. 1986.

21. SHANKER, S. et al. A longitudinal assessment of upper respiratory function and dentofacial morphology in 8 to 12 year-old children. Semin. Orthod., Philadelphia, v. 10, no. 1, p. 45-53, Mar. 2004.

22. SILVA FILHO, O. G. et al. Dimensões da nasofaringe em crianças de 7 anos de idade, portadoras de oclusão normal: avaliação pela cefalometria. Ortodontia, São Paulo, v. 2, n. 1, p. 20-30, jan./mar. 1989.

23. SUBTELNY, J. D. The significance of adenoid tissue in Orthodontia. Angle Orthod., Appleton, v. 24, no. 2, p. 59-69, Apr. 1954.

24. SUBTELNY, J. D. Oral respiration: facial maldevelopment and correcive dentofacial orthopedics. Angle Orthod., Appleton, v. 50, no. 3, p.147-164, July 1980.

25. TARVONEN, P. L.; KOSKI, K. Craniofacial skeleton of 7-year-old children with enlarged adenoids. Am. J. Orthod. Dentofacial Orthop., St. Louis, v. 91, no. 4, p. 300-304, Apr. 1987.

26. VALERA, F. C. P. et al. Muscular, functional and orthodontic changes in pre school children with enlarged adenoids and tonsils. Int. J. Pediatr. Otorhinolaryngol., Amsterdam, v. 67, p. 761-770, 2003.

27. VIG, P. S. et al. Quantitative evaluation of nasal airflow in relation to facial morphology. Am. J. Orthod., St. Louis, v. 79, no. 3, p. 263-272, Mar. 1981

28. WARREN, D. W.; LEHMAN, M. D.; HINTON, V. A. Analysis of simulated upper airway breathing. Am. J. Orthod., St. Louis, v. 86, no. 3, p. 197-206, Sept. 1984.

29. WARREN, D. W. et al. The relationship between nasal-oral breathing. Am. J. Orthod. Dentofacial Orthop., St. Louis, v. 93, no. 4, p. 289-293, Apr. 1988.

30. WARREN, D. W.; HAIRFIELD, W. M.; DALSTON, E. T. Effect of age on nasal cross-sectional area and respiratory mode in children. Laryngoscope, Philadelphia, v. 100, p. 89-93, Jan. 1990.

31. WATSON, R. M.; WARREN, D. W.; FISCHER, N. D. Nasal resistance, skeletal classification and mouth breathing in orthodontic patients. Am. J. Orthod., St. Louis, v. 54, no. 5, p. 367-379, May 1968.

32. ZANELATO, A. C. T. Determinação de medidas cefalométricas do espaço aéreo naso e bucofaríngeo em indivíduos com oclusão normal. 2003. 98 f. Tese (Mestrado Ortodontia)-Faculdade de Odontologia da Universidade Metodista de São Paulo, São Bernardo do Campo, 2003.
Endereço para correspondência
Aline Maria Alencar de Castro
Rua Vicente Leite, $\mathrm{n}^{\circ} 389$, apto. 900 - Meireles
CEP: $60.170-150$ - Fortaleza/CE
E-mail: alinemadc@yahoo.com.br 